\title{
Efficacy of interventions to reduce nosocomial transmission of SARS-CoV-2 in English NHS Trusts in Wave 1: A computational modelling study
}

Stephanie Evans ( $\sim$ stephanie.evans@phe.gov.uk)

UK Health Security Agency

Peter J White

Imperial College London

Mark Wilcox

Leeds Teaching Hospitals

Julie V Robotham

UK Health Security Agency

\section{Research Article}

Keywords: COVID-19, healthcare workers, patients, healthcare-associated infection, transmission, hospital

Posted Date: October 29th, 2021

DOI: https://doi.org/10.21203/rs.3.rs-1030531/v1

License: (9) This work is licensed under a Creative Commons Attribution 4.0 International License. Read Full License 


\section{Abstract}

Nosocomial transmission of SARS-CoV-2 has the potential to place a large burden on the healthcare system through, for example, increased patient length of stay, pressure on specialist care capacity and staff shortages. In England, a number of interventions were applied in hospitals over wave 1 of the COVID-19 pandemic to limit the spread of SARS-CoV-2 among both hospital inpatients and healthcare workers (HCWs). Using a computational modelling approach, we have estimated the combined effect of these, and other changes within the hospital setting, to determine how many nosocomial infections were averted. While highly uncertain, due primarily to important gaps in the evidence base, model results suggest that in a scenario with high occupancy, no testing, reduced infection prevention and control (IPC) measures, increased visitors, and longer patient stays, approximately $5.2 \%(3.9-7.2 \%)$ of all susceptible inpatients ( $\mathrm{n}=140,603 ; 95 \% \mathrm{Cl}, 89,352-197,977$ patients in total), and $51.1 \%(43.6,55.3 \%)$ of patientfacing HCWs could have been nosocomially infected with SARS-CoV-2 over wave 1 compared with the $1.0 \%(0.7,1.2 \%)$ of patients $(33,922 ; 24,089-41,015)$ and $20.3 \%(15.8-29.4 \%)$ of HCW observed to be nosocomially infected. The most effective interventions for prevention of nosocomial infections in patients were decreasing occupancy, increasing spacing between beds, and testing patients on admission, resulting in a reduction of $23,434(14,544,31,341), 10,979(2,458,16,979)$, and 9,505, $(4588$, 12,823 ) infections, respectively. Although every intervention had some impact, it was the collective impact of all interventions that demonstrated greatest effect, averting $140,603(89,352,197,977)$ infections in inpatients. In HCWs, the most effective intervention was universal mask use, with inclusion of universal masking as part of IPC measures averting 46.0\% (42.9-54.5\%) of infections in HCWs resulting in 17,980 $(2,772-28,450)$ fewer infections per 100,000 patient-facing HCWs. Interventions introduced over wave 1 of the SARS-CoV-2 pandemic in England reduced HCW infection rates by $51.1 \%$ (43.6-55.3\%).

\section{Introduction}

Over the course of the COVID-19 pandemic in England, there has been evidence of nosocomial transmission of the SARS-CoV-2 virus to both patients and healthcare workers (HCWs) [1-4], with 0.5-1\% of susceptible inpatients identified as developing a nosocomial infection between 01-March-2020 and 31August-2020 [1]. During this time period, several interventions were introduced in hospitals to reduce the transmission rate of SARS-CoV-2. These included regular testing of patients, increased cleaning and hand-hygiene, wearing of face masks/coverings universally, in addition to hospital system changes, such as reductions in occupancy and patients' length of stay, and socially distancing beds on wards $[5,6]$. Evidence around the efficacy of interventions such as wearing surgical masks or other respiratory protection equipment for SARS-CoV-2 is severely lacking. Although a number of studies exist for other respiratory viruses such as influenza or SARS-CoV-1, there is often disagreement about the magnitude of the effect observed between studies [7-13]. Arguably, the most reliable sources of information on efficacy of interventions come from a small number of systematic reviews or meta-analyses that combine the results of multiple studies to produce a combined estimate for the efficacy of individual interventions $[14,15]$. 
We present model-based estimates of the impact of a number of hospital-level interventions as part of a combined package for reducing the risk of nosocomial transmission of SARS-CoV-2. We also model the effect of changes in English hospitals in wave 1. We use a previously developed individual-based model of within-hospital transmission that has been calibrated against national datasets [16]. Using this model, we have previously demonstrated that for wave 1 , indirect patient-to-patient transmission was responsible for the majority of nosocomial SARS-CoV-2 infections in hospital inpatients in England; for infection acquisitions associated with work by HCWs, HCW-to-HCW transmission was the most common route [16], results that are supported by single site studies that integrate epidemiological and viral genomic data $[2,3]$.

We estimated the impact of eight interventions/changes that were implemented over wave 1 in hospitals in England. To explore the impact of these interventions and hospital changes on nosocomial infections in both inpatients and HCW, we: 1) combined evidence from the literature on the efficacy of individual measures for reducing the spread of respiratory viral infections in hospitals and the community; 2) modified the transmission probabilities and model parameters to reflect scenarios in which the interventions were 'reversed' (both individually and collectively); and then 3) modelled these counterfactual scenarios in which interventions were 'reversed', i.e. simulating the hypothetical scenario in which the interventions/changes had not been implemented, to estimate how much virus transmission they averted individually and when combined.

\section{Methods}

\section{Model development and calibration}

We have developed an individual-based model (IBM) of nosocomial transmission within and between patient and HCW populations. The model simulates transmission through different routes: i) patient-topatient transmission between those sharing a bay, ii) patient-to-patient transmission between patients residing on the same ward but not necessarily in the same bay, representing transmission through, for example, fomites, shared facilities, or healthcare workers acting as a vector for transmission, among others iii) patient-to-HCW transmission, iv) HCW-to-patient transmission, and v) HCW-to-HCW while present on the same ward. Infected patient cases are imported from the community at a rate calculated from observed hospital admissions in the NHSE Situation Report data [17]. HCWs can become infected in the community when they are outside of the hospital according to the predicted community prevalence on that date, where the prevalence was calculated from SPI-M estimates (SPI-M communication). Under the baseline scenario, the model is parameterised using multiple national datasets and literature, and is calibrated to reproduce the transmission dynamics of SARS-CoV-2 among healthcare workers (HCWs) and patients in an average English hospital in wave 1. A full model description and details of the calibration procedure are described in Supplementary File 1.

\section{Modelling the counterfactual by 'reversal' of interventions}


Counterfactual scenarios were simulated in which eight interventions were reversed (individually and collectively) and compared to a baseline, with intervention scenarios as follows: 1) Baseline transmission and interventions simulated to reflect actual numbers in first wave; 2) reversal of increased spacing between beds; 3 ) reversal of increased cleaning and hand-hygiene, 4) reversal of universal mask wearing by HCWs, 5) reversal of mask wearing by HCWs when treating patients, 6) reversal of decreased length of stay of admitted patients, 7) reversal of testing and cohorting of symptomatic patients on admission, 8) reversal of suspension of visitors to hospital patients; 9 ) reversal of decreased occupancy, 10) reversal of all interventions $2-9$ collectively.

To reverse interventions, available evidence for the efficacy of individual interventions was synthesised, and model parameters, specifically those describing routes of transmission (patient to patient, patient to $\mathrm{HCW}, \mathrm{HCW}$ to patient or HCW to HCW) were adjusted accordingly. Evidence on efficacy of interventions and data with which to adjust model parameters was drawn from multiple sources and was combined by randomly sampling values from an equally weighted combined distribution (Figure S1). Due to the paucity of literature evidence for the efficacy of mask wearing and hand-hygiene against SARS-CoV-2 transmission in a healthcare setting, confidence in the parameterisation of certain counterfactual scenarios varied (Figure 1, Table S1, S2). The interventions were reversed through adjustment of transmission parameters for each pathway according to the combined evidence (Figure S1). Intervention 6 (decreased length of stay of admitted patients) was reversed by modelling a scenario in which patients' lengths of stay reflected those prior to the COVID-19 pandemic. Age and sex adjusted length of stay of patients admitted to hospital between 01-Mar-2019 and April 2019 were calculated from data recorded in Secondary Uses Service data and using the $R$ library flexsurv [18]. Intervention 7 (testing and cohorting symptomatic patients on admission) was reversed by allowing patients to be admitted into any ward regardless of their SARS-CoV-2 status. Intervention 8 (suspension of visitors) was reversed by allowing up to two visitors per patient per day as described by [19], and assuming that each visitor stayed for an average of 14 minutes, constituting a transmission risk of 0.00125 per minute of visit $[20,21]$. Intervention 9 (decreased occupancy) was reversed by increasing modelled occupancy to reflect that observed in the NHS historically, allowing up to $85 \%$ of beds to be occupied [22].

After obtaining data for parameterising the reversal of interventions, new parameter sets were generated using the procedure described in Figure S1. First, 10 unique baseline transmission parameter sets were drawn from previous model calibrations, then for each intervention scenario 100 new parameters were generated by sampling 100 unique parameter values from the distribution of the combined study data; each set was mapped to one of the unique 10 transmission parameter sets to produce 100 new parameter sets Figure S1A. To reverse interventions where there was a single value, e.g. increased occupancy, each baseline transmission parameter set was replicated 10 times and then modified to include the single new parameter value in all sets (Figure S1B).

These new parameters were used within the IBM to generate estimates of efficacy of each intervention individually (in terms of nosocomial infections averted in inpatients and in HCW) as well as an overall estimate of the combined efficacy of all interventions that were implemented during wave 1 in England. 


\section{Simulations}

The model was simulated for 1110 time steps covering a time period of 185 days from 27-Feb-2020 to 31-Aug-2020 (6 steps per simulated day), covering wave 1 England. Individual-level patient and HCW data on infection status and location were recorded at each time step. Each parameter set generated in Reversal of interventions was simulated 5 times to generate both stochastic and parameter-based uncertainty.

Results are presented in terms of proportion of susceptible inpatients and proportion of the patient-facing HCW population becoming nosocomially infected with SARS-CoV-2 under each intervention scenario across the whole simulation period. For patients, the number of infections prevented is calculated from the simulated data. To scale up the single-hospital estimate to a national level, the total number of infections prevented is divided by the total number of beds in the simulated hospital (a proxy for hospital size), and then multiplied by the total number of beds across NHS hospitals in England using national data. This assumes that occupancy was similar between all trusts. For HCWs, the proportion of patientfacing staff becoming nosocomially infected are converted to estimates of numbers of infections prevented per 100,000 patient facing staff.

\section{Results}

\section{Efficacy of interventions on nosocomial infections in the patient population}

Under the baseline scenario, 1.0\% (median; IQR; $0.7-1.2 \%$ ) of all susceptible inpatients developed a nosocomial infection over wave 1 (Figure 2A). Over this time period, decreasing the length of stay was the single most effective change within hospitals for reducing risk to an admitted patient, and reversing this intervention increased the percentage of susceptible patients becoming infected to $1.4 \%(1.0-1.9 \%)$. Despite an increase in the percentage of susceptible patients developing a nosocomial infection when length of stay is increased, the total number of nosocomial infections in this scenario is lower than when other interventions are reversed due to the number of susceptible admissions also being reduced as a consequence of reduced patient turnover (Figure 2B, Figure S2). Increased cleaning and hand-hygiene, mask wearing by HCWs, testing patients on admission, and reducing occupancy were also effective interventions for reducing the percentage of susceptible patients that developed a nosocomial infection, and reversing any of these interventions increased the median proportion of patients developing a nosocomial infection to 1.1-1.2\% (0.9-1.6\%) (Figure 2A). In terms of reducing the absolute number of patients that were nosocomially infected, decreasing occupancy, increasing space between beds, and testing patients on admission were most effective with 23,434 (14,544-31,341), 10,979 (2,485-16,875), and 9,505 $(4,588-12,823)$ infections averted, respectively. It should be noted that the parameter describing the effectiveness of bed spacing is derived from only a single theoretical study, and so confidence in the effect of this measure is lower than for other interventions Table S1. 
Combined interventions had the greatest impact on reducing nosocomial spread; reversing all interventions in combination increased the proportion of patients developing a nosocomial infection to $5.2 \%$ (3.9-7.3\%). These results suggest that the combined effect of interventions that were in place during wave 1 of the SARS-CoV-2 pandemic in England averted up to $140,603(89,352,197,977)$ nosocomial infections in hospital inpatients (Figure 2B). The risk to patients of infection and the benefit of each intervention varied over time (Figure $2 \mathrm{C}, \mathrm{D}$ ). Testing patients on admission was more effective at the start of wave 1 when the number of infected patients and HCWs in hospital was at its highest level, whereas cleaning and hand-hygiene was most important in June when the number of susceptible patients peaked (Figure S3). Mask-wearing by HCWs played a similarly effective role at all times throughout the simulated period, but was slightly more effective in May and June when the number of infected HCWs peaked. No effect was observed for the intervention where visitors were re-introduced under the rules and assumptions included in this model.

\section{Efficacy of interventions on nosocomial infections in the HCW population}

Unlike the patient population, HCWs in the model are assumed to be able to become infected in both the hospital and community on a daily basis. Under baseline assumptions $21.3 \%(16.0,28.8)$ of HCWs were infected nosocomially between 01-March-2020 and 31-Aug-2020. Universal mask wearing by HCW played an important role in reducing HCW transmission events, and reversing mask wearing by HCWs universally resulted in a two-fold increase in HCW nosocomial infections, with 39.2\% (27.9 57.2) of HCWs becoming infected during wave 1 (Figure 3A). This equates to an additional 7,254 (4,605-12,669) infections for every 100,000 patient-facing HCWs (Figure 3B). Masking by HCWs prevented a substantial number of infections over the start of wave 1 (March - June). Notably, following the introduction of universal masking on 15th June, the greatest reduction was in HCW-to-HCW transmission, as suggested by the jump in averted infections in the latter half of wave 1 following the reversing of masking when not treating patients (Figure 3C,D).

\section{Discussion}

We have evaluated the impact of reversing any or all of eight interventions and changes occurring in NHS England hospitals over wave 1 of the COVID-19 pandemic on rates of nosocomial transmission to patients and HCWs in England. The literature search conducted to parameterise the model in this study highlighted a paucity of evidence around the efficacy of interventions, particularly increasing space between beds and of cleaning and hand-hygiene on reducing nosocomial transmission of respiratory viruses. Also, while several studies exist exploring the effect of mask-wearing, many of them are single site studies and their applicability outside of each setting is unclear (Table S1/S2). Furthermore, there is a lack of evidence around compliance with mask-wearing by healthcare workers when in communal areas. This is a key issue noting the relatively high prevalence of environmental contamination by SARS-CoV-2 in staff communal areas. There is additionally anecdotal evidence that HCW to HCW transmission may occur on travelling to/from work or between colleagues while socialising outside of work. 
While the baseline results are calibrated to high-quality national datasets, the scarcity of reliable evidence on the effectiveness of individual interventions (required for simulating a scenario in which they are reversed) is a key weakness of the results presented in this study. Additionally, while the model has been parameterised to best reflect the available data, uncertainty remains around the contribution of nosocomial and community sources of SARS-CoV-2 infection. As COVID-19 becomes an endemic disease and pressures on health systems from other seasonal respiratory pathogens increase, there is a critical need for evidence on the efficacy of such interventions on reducing nosocomial spread in order to design efficient and effective infection prevention and control strategies. We note also that the capacity to isolate patients in single rooms in English hospitals is limited. Approximately $80 \%$ of hospital beds are in multi-occupancy bays, and the remaining $20 \%$ are unevenly distributed within institutions, such that the great majority of patients (COVID-19 cases, unsuspected and suspected cases) were cohort nursed.

Due to the limited data available on effectiveness of interventions, further work will include conducting a formal elicitation of expert opinion alongside the simulation study. The elicitation exercise can be used to generate parameter distributions on, for example, compliance with and efficacy and feasibility of IPC measures, which can then be used to augment model inputs using evidence from literature, and allow further understanding on the uncertainty of model inputs and hence outputs.

While highly uncertain, given the dependency on (scant available) evidence on the effectiveness of interventions, model results suggest that in a scenario with high occupancy, no testing, reduced IPC, visitors, and longer stays, approximately $5.2 \%$ (3.9-7.0) of all susceptible inpatients $(140,603(89,352-$ $197,977)$ patients in total), and $44.1 \%$ of patient-facing HCWs could have been nosocomially infected with SARS-CoV-2 over wave 1 compared with the $1.0 \%$ of patients $(33,922(24,089-41,050)$ and $21.3 \%$ (16.0-28.8) of HCW observed. The most effective interventions and changes within hospitals for prevention of nosocomial infections in patients was decreased occupancy, increased spacing between beds, and testing patients on admission, resulting in prevention of $23,434(14,544-31,341), 10,979(2,458$ 16,979), and 9,505, (4588-12,823) nosocomial infections. However, every intervention evaluated had some impact in reducing transmission to inpatients, and it was the collective impact of all interventions that demonstrated greatest effect. In HCWs, the most effective single intervention was universal mask use, averting $46.0 \%$ (42.9-54.5\%) of infections (39.2 vs $21.3 \%)$, resulting in $17,980(2,772-28,450)$ fewer infections per 100,000 patient-facing HCWs. It is likely that the effect of this intervention was largely due to the dominance of the HCW-to-HCW transmission route $[2,16]$ but it is possible that there is also a cumulative effect of a reduction in HCW infections contributing to a reduction in HCW-to-patient transmission. There is evidence that the risk of patient-to-HCW and HCW-to-patient transmission events is small, presumably due to the efficacy of masking and hand-hygiene to prevent transmission $[2,3,16]$, but the potential for a larger effect of a small number of patient-to-HCW transmissions seeding larger outbreaks in the HCW population cannot be ignored.

A strength of the IBM is that it captures these 'knock-on' benefits associated with prevention of transmission chains both within and across patient and HCW populations; such combined/bundled effects are sometimes referred to as the 'Swiss Cheese' infection prevention model $[23,24]$. This 
cumulative effect of reducing transmissions is apparent when looking at the impact of removing all interventions in combination, which results in a higher number of nosocomial infections than the sum of the individual interventions. Model findings suggest that collectively the interventions introduced over wave 1 of the SARS-CoV-2 pandemic in England averted 140,603 (89,352-197,977) infections in inpatients and reduced HCW infection rates by $51.1 \%$ (43.6-55.3\%).

Previous modelling studies have shown that HCW to HCW transmission was a dominant route of transmission within hospitals in wave 1 of the pandemic $[16,25,26]$, and impacts transmission dynamics to the greatest degree. Universal mask wearing by HCWs is an intervention which impacts this route. The dominance of this transmission route, combined with the evidence of effectiveness of universal mask wearing being high, results in high impact of universal mask wearing by HCW. By evaluating the literature, we estimate that universal mask-wearing by HCWs with complete compliance reduces the probability of transmission by a factor of 1.26-4, with a median effect of $3.2[7,14]$. However, there is evidence that $\mathrm{HCW}$ do not perceive interactions with other HCWs as a potential risk for becoming infected with SARSCoV-2 [27], and that there are certain times that preventative measures such as mask-wearing and social distancing are not possible (e.g. while eating in a shared communal area) [28, 29]. Both of these factors, and others, could reduce compliance with the guidance updated on June 15th 2020 stating that masks/face coverings should be worn universally (i.e. by HCWs, patients and visitors). We assumed that HCWs fully complied with universal masking upon the change in guidance, wearing masks for their entire shift and for every interaction with patients and other $\mathrm{HCWs}$, thus the reduction in transmission resulting from wearing a mask was applied to every HCW interaction. If this was not the case the modelled impact of mask wearing around other HCWs would fall as the reduction in transmission would only be applied to those interactions where masks were worn. The impact of mask-wearing by patients was not included in the model, however implementation of masking in patients was poor during Summer 2020, i.e. throughout wave 1.

When HCWs wore masks only when treating patients and not for contacts with other HCWs (prior to June), the number of infections increased by approximately $30 \%$ (from $21.32(15.95,28.78)$ to $26.4 \%$ (18.7-41.5)). Evidence from a single site study between 01-Mar-2020 and 25-Jul-2020 suggested, that $50 \%$ of $\mathrm{HCW}$ infections were nosocomial, and $55.3 \%$ of non-imported cases were likely to be attributable to HCW-to-HCW transmission [2]. The authors of this paper also examined samples from the second wave (30-Nov-2020 - 24-Jan-2021) that followed the change in guidance, and thus for this time period HCWs were advised to wear face-coverings in all areas. During this period of study the proportion of infections that were acquired in the community remained at $50 \%$, but the proportion attributable to $\mathrm{HCW}$ to $\mathrm{HCW}$ transmission fell to $37.4 \%$, i.e. there was a reduction by a factor of 1.48 . An additional study in a Parisian hospital that was not included in the initial model parameterisation estimated that the adjusted odds ratio for HCWs that did not wear masks over the period of study (in wave 1) was 13.1; however, there was a large amount of uncertainty around this estimate [30]. These studies are largely consistent with our estimate that over wave 1 an additional 22.7\% (12.3-35.7\%) of HCWs would have been infected had the guidance on mask wearing not changed. 
In previous work using the same IBM as used here, we have shown that patient-to-patient transmission is the dominant source of transmission to hospital inpatients $[16,25]$. This has been supported by single centre genomic studies. One of these showed that of the non-imported cases identified in patients, $63.6 \%$ of infections in wave 1 and $79.2 \%$ of infections in wave 2 were attributable to patient-to-patient transmission [2]. Another study showed that transmission outside of a ward is unlikely to be a major contributor to nosocomial outbreaks in hospital inpatients [3]. It is therefore expected that interventions that reduce the risk of transmission to patients sharing a bay or ward with infected patients will be the most effective in reducing transmission. We predict that the most effective interventions for reducing the risk of transmission to patients (i.e. those that had the greatest effect when they were removed), were increasing spacing between beds, increasing hand-hygiene of HCWs, decreasing the length of stay of inpatients, and testing on admission. A single trust study that compared infection rates before and after increasing bed spacing also found a significant impact of increasing space between beds in terms of reducing nosocomial SARS-CoV-2 transmission [31].

It should be noted that these findings are estimates for the impact of hospital interventions on nosocomial infections in patients and HCW for wave 1 of the COVID-19 pandemic only. The impacts of each of these components of control could have likely changed in subsequent waves, noting, for example, alterations in the transmissibility of virus variants, increased capacity for rapid testing upon hospital admission, and changes in host immunity, including once vaccination was introduced.

There are several limitations of this work, most importantly the aforementioned reliance on limited evidence of individual intervention effectiveness. To be transparent about the provenance and quality of the data used we have categorised effectiveness estimates according to the study type and pathogen of study. However, lack of evidence means that conclusions should be treated with caution. Several simplifying assumptions have been made in the model, including equal risk across all patients and patient-facing HCW when interacting with infected patients or HCWs, interventions being applied uniformly across the hospital and across the simulation period. It is also of note that estimates of intervention effectiveness reflect the level of compliance with the interventions in the particular studies, which was not reported. We do not adjust for compliance in our model, and use the direct estimate of efficacy reported in the literature in our simulations. If compliance in the reported studies differed from that exhibited in practice, the impact of the intervention on nosocomial transmission would change in accordance. Reversing of interventions only considered those described, additional measures such a double gloving or sessional gown use are not included in this work.

This study uses previously published parameter estimates on the efficacy of individual measures to evaluate the contribution individual interventions to the reduction of nosocomial transmission as part of the collection of interventions and hospital changes in place in wave 1. A strength of the modelling approach used here is the ability of the model to capture cumulative effects of interventions through reducing the seeding of new infection clusters. These results highlight the importance of maintaining high levels of compliance to infection prevention and control measures in hospitals and have important implications as hospitals prepare for a surge in demand due to emerging winter pressures and COVID-19. 


\section{Declarations}

\section{Funding}

SE and JR are jointly funded by UKRI and NIHR [COV0357; MR/V028456/1]. PJW was supported by the MRC Centre for Global Infectious Disease Analysis (grant MR/R015600/1), which is jointly funded by the UK Medical Research Council (MRC) and the UK Foreign, Commonwealth \& Development Office (FCDO), under the MRC/FCDO Concordat agreement and is also part of the EDCTP2 programme supported by the European Union (EU). PJW was also supported by the National Institute for Health Research (NIHR) Health Protection Research Unit (HPRU) in Modelling and Health Economics, a partnership between the UK Health Security Agency (UKHSA), Imperial College London and LSHTM (grant NIHR200908).

\section{Competing interest statement}

PJW has received payment from Pfizer for teaching of mathematical modelling of infectious disease transmission and vaccination.

\section{Disclaimer}

The views expressed are those of the authors and not necessarily those of the Department of Health and Social Care, EU, FCDO, MRC, National Health Service, NIHR, or UKHSA.

\section{Ethical Approval}

Ethical approval was not required for this study

\section{References}

1. Bhattacharya A, Collin SM, Stimson J, Thelwall S, Nsonwu O, Gerver S, et al. Healthcare-associated COVID-19 in England: a national data linkage study. medRxiv. 2021 Feb 19;2021.02.16.21251625.

2. Lindsey BB, Villabona-Arenas CJ, Campbell F, Keeley AJ, Parker MD, Shah DR, et al. Characterising within-hospital SARS-CoV-2 transmission events: a retrospective analysis integrating epidemiological and viral genomic data from a UK tertiary care setting across two pandemic waves. medRxiv. 2021 Jul 19;2021.07.15.21260537.

3. Illingworth CJ, Hamilton WL, Warne B, Routledge M, Popay A, Jackson C, et al. Superspreaders drive the largest outbreaks of hospital onset COVID-19 infections. Walczak AM, Ogbunugafor CB, Cobey SE, editors. eLife. 2021 Aug 24;10:e67308.

4. Ellingford JM, George R, McDermott JH, Ahmad S, Edgerley JJ, Gokhale D, et al. Genomic and healthcare dynamics of nosocomial SARS-CoV-2 transmission. Elife. 2021 Mar 17;10.

5. May R, Powis S. Expansion of patient testing for Covid-19. 2020.

6. COVID-19: infection prevention and control (IPC) [Internet]. GOV.UK. [cited 2021 Mar 12]. Available from: https://www.gov.uk/government/publications/wuhan-novel-coronavirus-infection-prevention- 
and-control

7. Cheng Y, Ma N, Witt C, Rapp S, Wild PS, Andreae MO, et al. Face masks effectively limit the probability of SARS-CoV-2 transmission. Science. 2021 May 20;

8. Kua J, Patel R, Nurmi E, Tian S, Gill H, Wong DJN, et al. healthcareCOVID: a national cross-sectional observational study identifying risk factors for developing suspected or confirmed COVID-19 in UK healthcare workers. PeerJ. 2021 Feb 4;9:e10891.

9. Loeb M, McGeer A, Henry B, Ofner M, Rose D, Hlywka T, et al. SARS among Critical Care Nurses, Toronto. Emerg Infect Dis. 2004 Feb;10(2):251-5.

10. Nishiyama A, Wakasugi N, Kirikae T, Quy T, Ha LD, Ban VV, et al. Risk factors for SARS infection within hospitals in Hanoi, Vietnam. Jpn J Infect Dis. 2008 Sep;61(5):388-90.

11. Raboud J, Shigayeva A, McGeer A, Bontovics E, Chapman M, Gravel D, et al. Risk Factors for SARS Transmission from Patients Requiring Intubation: A Multicentre Investigation in Toronto, Canada. PLOS ONE. 2010 May 19;5(5):e10717.

12. Seto W, Tsang D, Yung R, Ching T, Ng T, Ho M, et al. Effectiveness of precautions against droplets and contact in prevention of nosocomial transmission of severe acute respiratory syndrome (SARS). Lancet. 2003 May 3;361(9368):1519-20.

13. Teleman MD, Boudville IC, Heng BH, Zhu D, Leo YS. Factors associated with transmission of severe acute respiratory syndrome among health-care workers in Singapore. Epidemiol Infect. 2004 Oct;132(5):797-803.

14. Howard J, Huang A, Li Z, Tufekci Z, Zdimal V, van der Westhuizen H-M, et al. An evidence review of face masks against COVID-19. Proc Natl Acad Sci U S A. 2021 26;118(4).

15. Wong VWY, Cowling BJ, Aiello AE. Hand hygiene and risk of influenza virus infections in the community: a systematic review and meta-analysis. Epidemiol Infect. 2014 May;142(5):922-32.

16. Evans S, Stimson J, Pople D, Robotham J. Quantifying the contribution of pathways of nosocomial acquisition of COVID-19 in English hospitals. (In press) International Journal of Epidemiology. 2021;

17. Digital NHS. Hospital Episode Statistics (HES): Provisional Monthly Statistics for Admitted Patient Care, Outpatient and Accident \& Emergency [Internet]. 2018 [cited 2020 May 18]. Available from: https://data.gov.uk/dataset/c914f943-98d1-4dee-aba5-c40a7d08eefa/hospital-episode-statisticshes-provisional-monthly-statistics-for-admitted-patient-care-outpatient-and-accident-emergency

18. Jackson C. flexsurv: A Platform for Parametric Survival Modeling in R. Journal of Statistical Software. 2016 May 12;70(1):1-33.

19. Scaria E, Barker AK, Alagoz O, Safdar N. Association of Visitor Contact Precautions With Estimated Hospital-Onset Clostridioides difficile Infection Rates in Acute Care Hospitals. JAMA Netw Open. $202101 ; 4(2): e 210361$.

20. Cohen B, Hyman S, Rosenberg L, Larson E. Frequency of Patient Contact with Health Care Personnel and Visitors: Implications for Infection Prevention. Jt Comm J Qual Patient Saf. 2012 Dec;38(12):560-5. 
21. Temime L, Gustin M-P, Duval A, Buetti N, Crépey P, Guillemot D, et al. A Conceptual Discussion About the Basic Reproduction Number of Severe Acute Respiratory Syndrome Coronavirus 2 in Healthcare Settings. Clin Infect Dis. 2021 23;72(1):141-3.

22. NHS England. Statistics» Bed Availability and Occupancy Data [Internet]. [cited 2020 Feb 17]. Available from: https://www.england.nhs.uk/statistics/statistical-work-areas/bed-availability-andoccupancy/bed-data-day-only/

23. Noh JY, Song JY, Yoon JG, Seong H, Cheong HJ, Kim WJ. Safe hospital preparedness in the era of COVID-19: The Swiss cheese model. Int J Infect Dis. 2020 Sep;98:294-6.

24. Yanke E, Carayon P, Safdar N. Translating evidence into practice using a systems engineering framework for infection prevention. Infect Control Hosp Epidemiol. 2014 Sep;35(9):1176-82.

25. Evans S, Agnew E, Vynnycky E, Stimson J, Bhattacharya A, Rooney C, et al. The impact of testing and infection prevention and control strategies on within-hospital transmission dynamics of COVID-19 in English hospitals. Philosophical Transactions of the Royal Society B: Biological Sciences. $2021 \mathrm{Jul}$ 19;376(1829):20200268.

26. Mo Y, Eyre DW, Lumley SF, Walker TM, Shaw RH, O'Donnell D, et al. Transmission dynamics of SARSCoV-2 in the hospital setting. medRxiv. 2021 May 1;2021.04.28.21256245.

27. Brandt MP, Jäger W, Epple S, Haferkamp A, Schröder A. SARS-CoV-2 outbreak in medical employees in a large urologic department: Spread, containment and outcome. Am J Infect Control. 2021 Jun;49(6):674-7.

28. Richterman A, Meyerowitz EA, Cevik M. Hospital-Acquired SARS-CoV-2 Infection: Lessons for Public Health. JAMA. 2020 Dec 1;324(21):2155-6.

29. Final report - Healthcare Safety Investigation Branch [Internet]. [cited 2020 Dec 7]. Available from: https://www.hsib.org.uk/investigations-cases/covid-19-transmission-hospitals/final-report/

30. Davido B, Gautier S, Riom I, Landowski S, Lawrence C, Thiebaut A, et al. The first wave of COVID-19 in hospital staff members of a tertiary care hospital in the greater Paris area: A surveillance and risk factors study. Int J Infect Dis. 2021 Apr;105:172-9.

31. Scientific Advisory Group for Emergencies. $\mathrm{HOCl}$ and EMG. Masks for healthcare workers to mitigate airborne transmission of SARS-CoV-2. 2021.

32. Clapp PW, Sickbert-Bennett EE, Samet JM, Berntsen J, Zeman KL, Anderson DJ, et al. Evaluation of Cloth Masks and Modified Procedure Masks as Personal Protective Equipment for the Public During the COVID-19 Pandemic. JAMA Intern Med. 2021 01;181(4):463-9.

33. Recovery position [Internet]. [cited 2021 Aug 4]. Available from: https://nhsproviders.org/recoveryposition-what-next-for-the-nhs/constraints-and-considerations-for-resuming-services

34. Martos DM, Parcell BJ, Eftimie R, Martos DM, Parcell BJ, Eftimie R. Modelling the transmission of infectious diseases inside hospital bays: implications for COVID-19. MBE. 2020;17(6):8084-104.

35. Bhattacharya A, Collin SM, Stimson J, Thelwall S, Nsonwu O, Gerver S, et al. Healthcare-associated COVID-19 in England: A national data linkage study. J Infect. 2021 Aug 30; 
36. COVID-19 infection prevention and control guidance.:49.

37. Martos DM, Parcell BJ, Eftimie R. Modelling the transmission of infectious diseases inside hospital bays: implications for Covid-19. medRxiv. 2020 Sep 8;2020.09.04.20188110.

38. Wong SCY, Kwong RT-S, Wu TC, Chan JWM, Chu MY, Lee SY, et al. Risk of nosocomial transmission of coronavirus disease 2019: an experience in a general ward setting in Hong Kong. J Hosp Infect. 2020 Jun;105(2):119-27.

\section{Figures}

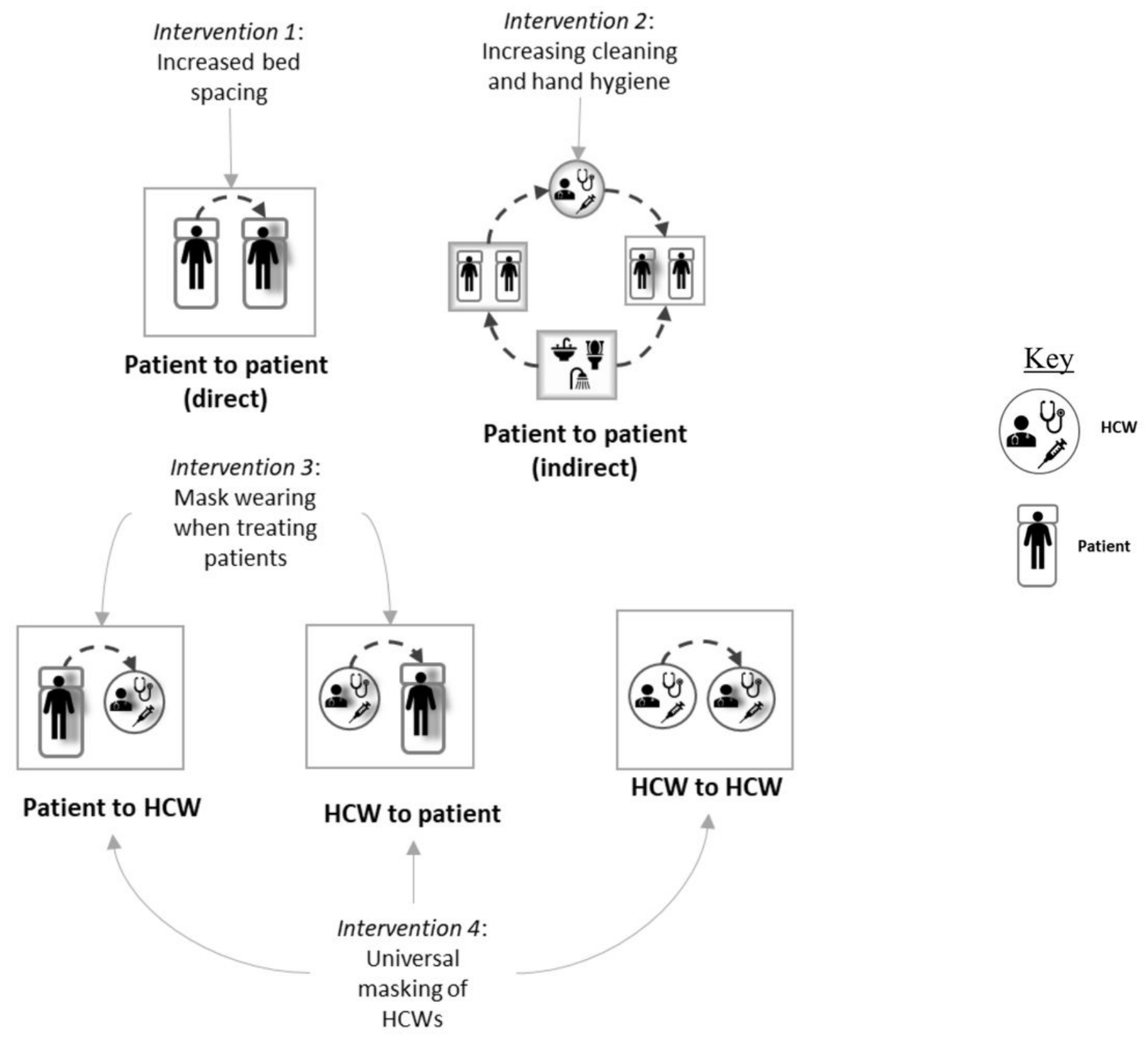

Figure 1 
Schematic of interventions. Dashed arrows represent transmission routes, solid grey arrows indicate the transmission routes the intervention has an impact upon.
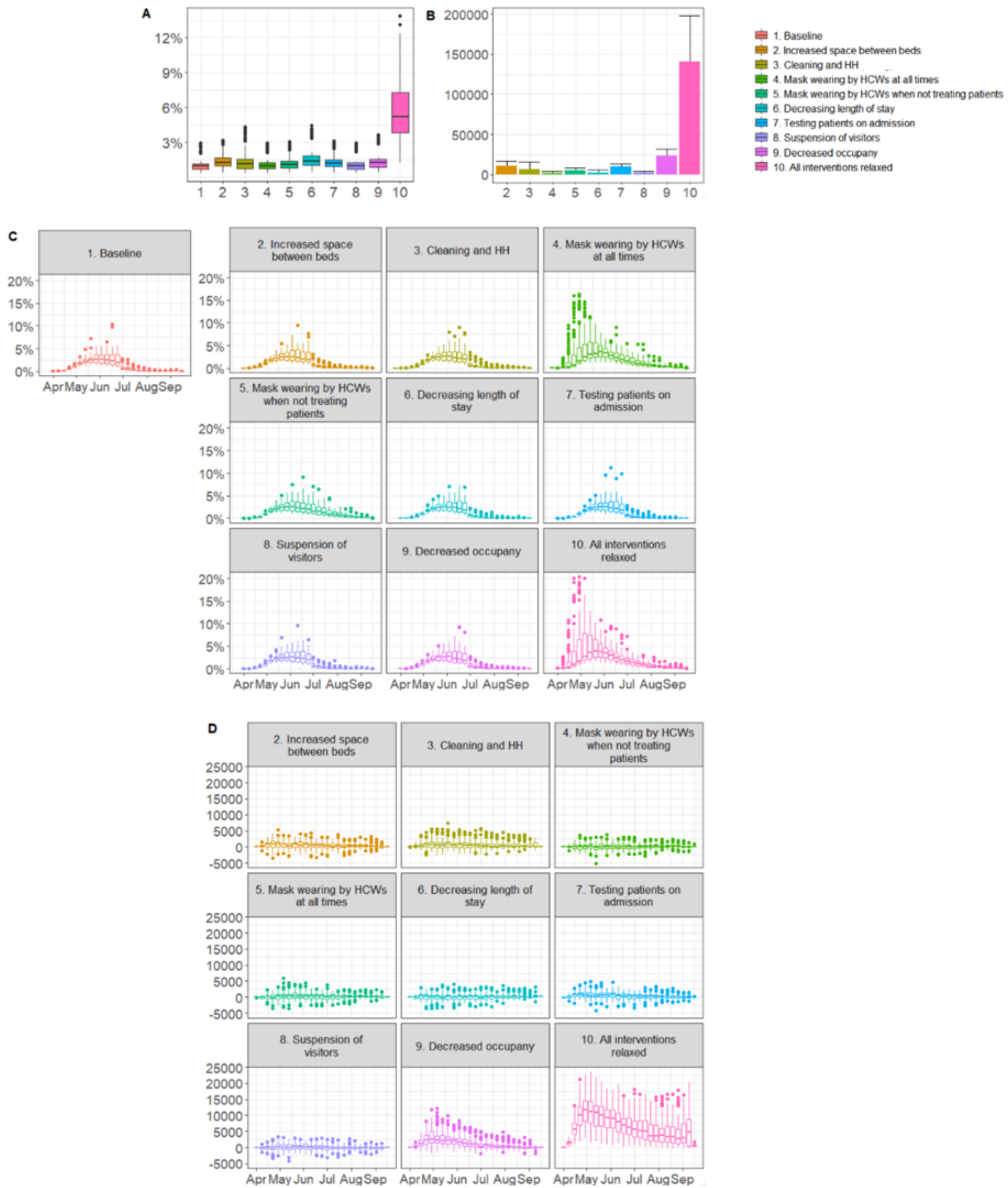

\section{Figure 2}

Effect of relaxing single or combined interventions on nosocomial acquisition rates in hospital inpatients. A) Proportion of susceptible inpatients infected when individual (1-9) or combined (10) interventions are 
lifted. B) Number of nosocomial infections averted by adding each intervention into the package of all interventions. C) Percentage of patients infected per week. D) Infections averted per week.
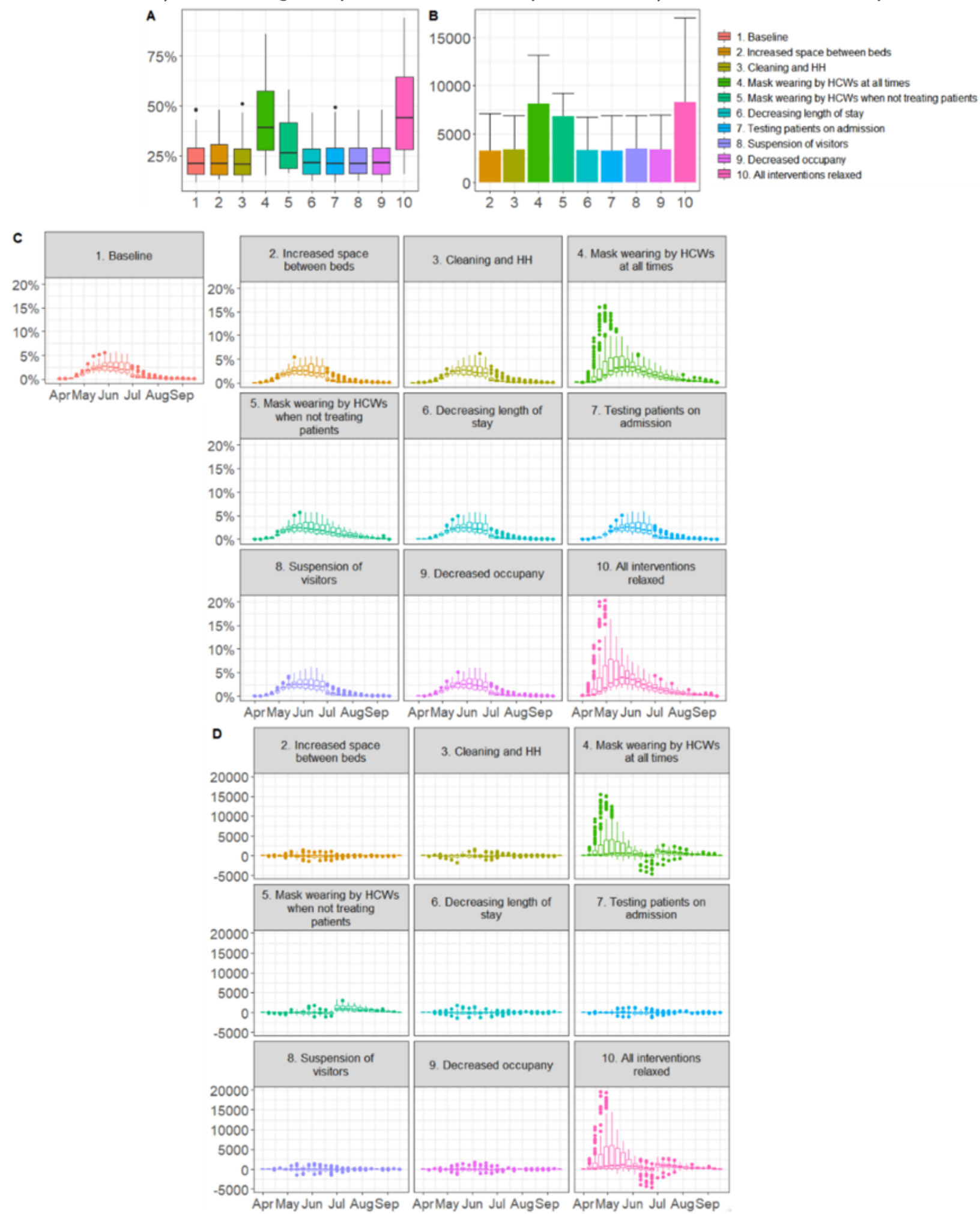

\section{Figure 3}

Effect of relaxing single or combined interventions on SARS-CoV-2 infection rates in patient-facing HCWs. A) Proportion of HCWs infected when individual (1-9) or combined (10) interventions are lifted. B) 
Number of patient-facing HCW infections averted by adding each intervention into the package of all interventions. C) Percentage of HCWs infected per week. D) Infections averted per week.

\section{Supplementary Files}

This is a list of supplementary files associated with this preprint. Click to download.

- InterventionsW1suppfile1.docx

- SupplementaryFiguresandTables.docx 